\title{
Recontacting clinical genetics patients with reclassified results: equity and policy challenges
}

\author{
Yvonne Bombard $^{1,2} \cdot$ Chloe Mighton $^{1,2}$
}

Published online: 19 December 2018

(c) European Society of Human Genetics 2018

The growing use of sequencing approaches will not only increase the volume of variants identified but also those that will be subsequently reclassified and may warrant patient recontact. However, there is limited guidance on if or when patients should be recontacted. Inconsistencies persist in how recontacting is carried out within and across jurisdictions. These are compounded by an overall lack of clarity about which professionals are responsible for recontacting and concerns about the resources and infrastructure required to achieve recontacting in a sustainable manner $[1,2]$. Thus, Carrieri et al.'s [3] recommendations for recontacting patients offer a timely set of evidence-based $[1,2,4]$ principles that address a critical gap in practice and policy.

Carrieri et al. conclude that recontacting is a shared responsibility between laboratories, patients, and providers, desirable in situations where findings have clinical or personal utility, though there is no clinical "duty" to do so. The recommendations state that each country should determine its own methods to operationalize recontacting, cautioning that recontacting should be equitable, and sustainable for the healthcare system. These recommendations are an important contribution to the field. Nonetheless, the latter caveats raise some significant challenges that ought to be considered.

One major consideration is equity. Diversity in the organization, delivery, regulation and funding of healthcare systems, and genetics services across Europe will necessarily lead to variation in recontacting practices across jurisdictions. Indeed, as recontacting is not deemed a duty, it is ultimately at the discretion of the genetics service or

Yvonne Bombard

Yvonne.Bombard@utoronto.ca

1 Institute of Health Policy, Management and Evaluation, University of Toronto, Toronto, ON, Canada

2 Li Ka Shing Knowledge Institute, St. Michael's Hospital, Toronto, ON, Canada providers to determine if and when to recontact patients with reinterpreted variants. Different genetics services and providers may choose to recontact patients for different reasons, for different variant reclassifications, and through different modes of communication-practice variation which already exists based on the authors' European survey [4]. This has the potential to result in inequities in access to information, and ultimately, inequities in outcomes.

Inequities already exist across ethnic groups due to under-representation of genomes from non-European populations in current databases that collate pathogenicity of sequence variants. This has resulted in a significantly lower understanding of variant pathogenicity in these underrepresented populations compared to European populations. Indeed, rates and types of variant reclassifications differ between patients of different ancestries [5]. Patients of non-European descent are more likely to harbor variants of uncertain significance (VUS) [6], which are generally reclassified more often than other variants [5], and are also more likely receive misclassified variants such as pathogenic variants that should have been classified as benign [7]. Any variations that arise related to recontacting may therefore disproportionately disadvantage these patients. As genomic databases diversify, our ability to interpret the sequences of non-European individuals will improve. If we do not create more comprehensive databases and uniform policies for recontacting, we reinforce, and likely exacerbate, existing health disparities.

The opportunity costs of recontacting also deserve attention. Recontacting can be time-intensive and resourceintensive [8]; requiring dedicated resources for laboratories to store and reinterpret genomic data as well as ongoing resources for clinicians to identify, track, communicate with, and manage patients whose results have been updated. In publicly funded healthcare systems, allocating resources towards recontacting inherently necessitates allocating resources away for other areas of clinical practice, potentially to the detriment of patient care. There is a paucity of evidence on the economic and health system impacts 
associated with recontact. This is a critical gap given that healthcare systems already operate within constrained budgets with limited resources available for genetics services. At the very least, this evidence will be essential to ensure that recontacting is sustainable, if not equitable.

The distinctions between the concepts of clinical and personal utility are gaining traction in genomics practice and policy [9]. Indeed, Carrieri et al. recommend that recontact is desirable for results with clinical or personal utility. However, clinical and personal utility may be interpreted and defined in different ways by providers, which can diverge from patients' perceptions of utility [10]. Without consensus on how "utility" and its thresholds should be defined, there is the potential for inequities in information provision and subsequent outcomes. For instance, many variant reclassifications are downgrades of VUS [5]. Given that management of VUS should be based on personal and family history, not the VUS, this type of reclassification may not impact a patient's medical care, and may not have sufficient clinical utility to warrant recontact. However, the alleviation of ambiguity related to VUS may have personal utility or psychosocial benefits for the patient and their family members. More evidence and guidance is needed on what scenarios provide sufficient clinical and/or personal utility to justify recontact.

Consent remains an illusive but vital consideration. The extent to which patients' preferences should determine the types of information they are recontacted about is unclear. Additionally, should patients should provide consent for recontacting at one time point or update their preferences over time? This is critical in light of survey findings that providers override patients' preferences and return updated results even when patients indicate they do not wish to be recontacted [4].

Carrieri et al.'s evidence-based recommendations are an important step towards addressing the limited guidance that exists on recontact in the clinical setting. Given the paucity of guidance on recontacting research participants with reinterpreted results, Carrieri et al.'s recommendations may also inform approaches for recontacting in the research setting. We anticipate that recommendations across both clinical and research contexts will evolve as we acquire more experience and evidence to inform them. The onus is now on our community to build that evidence-base to ensure equitable and sustainable recontacting practices.

\section{References}

1. Carrieri D, Dheensa S, Doheny S, et al. Recontacting in clinical practice: an investigation of the views of healthcare professionals and clinical scientists in the United Kingdom. Eur J Hum Genet. 2017;25:275-9.

2. Carrieri D, Dheensa S, Doheny S, et al. Recontacting in clinical practice: the views and expectations of patients in the United Kingdom. Eur J Hum Genet. 2017;25:1106-12.

3. Carrieri D, Howard H, Benjamin C, et al. Recontacting patients in clinical genetics services: recommendations of the European Society of Human Genetics. Eur J Hum Genet. 2018. https://doi. org/10.1038/s41431-018-0285-1 [Epub ahead of print]

4. Sirchia F, Carrieri D, Dheensa S, et al. Recontacting or not recontacting? A survey of current practices in clinical genetics centres in Europe. Eur J Hum Genet. 2018;26:946-54.

5. Slavin TP, Van Tongeren LR, Behrendt CE, et al. Prospective study of cancer genetic variants: variation in rate of reclassification by ancestry. J Natl Cancer Inst. 2018;110:1059-66.

6. Finch A, Wang M, Fine A, et al. Genetic testing for BRCA1 and BRCA2 in the province of Ontario. Clin Genet. 2016;89:304-11.

7. Manrai AK, Funke BH, Rehm HL, et al. Genetic misdiagnoses and the potential for health disparities. $\mathrm{N}$ Engl $\mathrm{J}$ Med. 2016;375:655-65.

8. Beunders G, Dekker M, Haver O, Meijers-Heijboer HJ, Henneman L. Recontacting in light of new genetic diagnostic techniques for patients with intellectual disability: feasibility and parental perspectives. Eur J Med Genet. 2018;61:213-8.

9. Bombard Y, Bach PB, Offit K. Translating genomics in cancer care. J Natl Compr Canc Netw. 2013;11:1343-53.

10. Jamal L, Robinson JO, Christensen KD, et al. When bins blur: patient perspectives on categories of results from clinical whole genome sequencing. AJOB Empir Bioeth. 2017;8:82-8. 